ACCEPTED MANUSCRIPT

\title{
Mechanisms of Friction Reduction of Nanoscale Sliding Contacts Achieved Through Ultrasonic Excitation
}

To cite this article before publication: Hossein Jiryaei Sharahi et al 2018 Nanotechnology in press https://doi.org/10.1088/1361-6528/aaf3cd

\section{Manuscript version: Accepted Manuscript}

Accepted Manuscript is "the version of the article accepted for publication including all changes made as a result of the peer review process, and which may also include the addition to the article by IOP Publishing of a header, an article ID, a cover sheet and/or an 'Accepted

Manuscript' watermark, but excluding any other editing, typesetting or other changes made by IOP Publishing and/or its licensors"

This Accepted Manuscript is @ 2018 IOP Publishing Ltd.

During the embargo period (the 12 month period from the publication of the Version of Record of this article), the Accepted Manuscript is fully protected by copyright and cannot be reused or reposted elsewhere.

As the Version of Record of this article is going to be / has been published on a subscription basis, this Accepted Manuscript is available for reuse under a CC BY-NC-ND 3.0 licence after the 12 month embargo period.

After the embargo period, everyone is permitted to use copy and redistribute this article for non-commercial purposes only, provided that they adhere to all the terms of the licence https://creativecommons.org/licences/by-nc-nd/3.0

Although reasonable endeavours have been taken to obtain all necessary permissions from third parties to include their copyrighted content within this article, their full citation and copyright line may not be present in this Accepted Manuscript version. Before using any content from this article, please refer to the Version of Record on IOPscience once published for full citation and copyright details, as permissions will likely be required. All third party content is fully copyright protected, unless specifically stated otherwise in the figure caption in the Version of Record.

View the article online for updates and enhancements. 
Keywords: Ultrasonic force microscopy, Friction, Wear, Nanotribology, Polystyrene

\begin{abstract}
Friction reduction is an important issue for proper functioning of nano/micro-electromechanical systems (N-/MEMS) due to their large surface to volume ratios and the inability of traditional liquid lubricants to effectively lubricate sliding contacts. One efficient technique to achieve substantially lowered friction at nanoscale, as well as superlubricity in some instances, was investigated with the coupling of ultrasonic actuation of the sliding contact in an atomic force microscope (AFM). Despite the successful application of ultrasonic AFM methods in achieving mechanical property measurements and nanoscale subsurface imaging of soft and hard materials, the mechanism of friction reduction in the microscopic contact and the influence of the ultrasonic parameters on friction reduction are still elusive. In this study, the effects of excitation amplitude, applied normal force, tip radius, and humidity on friction have been investigated in detail. Ultrasonic force microscopy (UFM) results are compared against those collected with conventional contact-AFM (C-AFM) and indicate that a reduction in the adhesive interaction between the tip and sample, as well as a reduction in the shear strength can explain the mechanisms of the friction reduction in UFM method. This study opens up a new door for the control of friction and wear, which is critical for the increased lifetime of AFM probes, N-/MEMS devices and would potentially bridge the gap between nanotribology and other fields, such as nanomachining, nanolithography and biomaterials imaging.
\end{abstract}

\title{
1. Introduction
}

The study of friction and wear at the nanoscale is of tremendous significance in a diverse range of mechanical systems, such as in nano-/microelectromechanical systems (N-/MEMS), polymer coatings, and nanoscale biological imaging [1, 2, 3]. The atomic force microscope (AFM) [4] and surface force apparatus (SFA) [5], have opened a way for the fundamental study of friction and wear phenomena in nano-/microscale. These tools showed that the macroscopic laws of friction do not always apply in nano-/microscopic systems because of their large surface to volume ratio, greater 


\section{Nanotechnology}

effects of surface chemistry, adhesion, and surface roughness[1]. On the other hand, control of friction and wear is a great deal of interest for increasing the lifetime of $\mathrm{N}$ /MEMS, controlling the forces in nanomachining and nanolithography, and enhancing the resolution of biomaterials imaging $[6,7]$. Conventional mechanisms of controlling friction and wear are achieved through chemical modification of the surfaces at the sliding interface, typically by applying base lubricants with friction modifier additives [1]. For example, electrospun polymer microbeads are added to a fluid lubricant (mineral oil) to reduce friction [8]. These traditional lubrication techniques are ineffective at the nanometer scale since there is a significant increase in the their viscosity when molecular chains are confined into nanometer-sized gaps [9, 1]. Additionally, surface texturing methods based on laser ablation, electrical discharge and focused ion beam milling have been used to create surfaces that can exhibit lower friction coefficients, however these methods are destructive and invasive [10, 11].

Recently, actuation of the sample at ultrasonic frequencies/during scanning with an AFM has allowed for the characterization of the mechanical properties of the surface and subsurface features $[12,13,14]$. It has also been observed that these AFM techniques have shown the ability to reduce friction and wear in nanoscale sliding contacts $[9,15,16,17]$. Ultrasonic force microscopy (UFM) is one such example [18]. In this case, a sample is actuated at ultrasonic frequencies using a piezoelectric actuator beneath the sample. At sufficiently high sample actuation frequencies (typically $\mathrm{MHz}$ frequencies), far away from the resonance frequency of the cantilever, the ultrasonic displacement of the cantilever carries information about elastic and adhesive properties of surface and subsurface features $[14,19]$. This method also showed the friction reduction on hard materials, such as silicon wafers and glass substrates, when a silicon AFM tip is slid against them $[16,20]$. Another available technique for imaging the buried nanoscale features and control of the friction is atomic force acoustic microscopy (AFAM), which has been implemented to measure contact stiffness and reduce the friction by exciting the sample or cantilever near contact resonance frequencies [21, 9]. To show the effects of ultrasonic vibration on friction reduction on soft sample, AFAM has been applied to reduce the formation of periodic ripples on a polymeric thin film [9]. Since the cantilever is excited at contact resonance frequency in this method, it causes some issues. For example, contact resonance frequency is affected by the mechanical stiffness of the sample, sample topography, and the normal force on the tip. Therefore, interpreting of friction forces is very challenging in this method. In UFM method, the undesirable effects of the resonance will be minimized, since the samples are excited at frequency much higher than the contact resonance frequency of the cantilever.

Although the mechanisms of ultrasonic AFM in subsurface imaging have been studied theoretically and experimentally in detail $[22,12]$, the exact mechanism of fiction reduction and the influence of the ultrasonic parameters on friction and wear reduction are still unknown. It has been proposed that ultrasonic vibration with sufficiently high amplitude can replace a traditional lubricant in nanoscale contacts, as friction vanishes when the tip-sample contact breaks for part of the vibration cycle [16]. Another 


\section{Nanotechnology}

mechanism of friction reduction explains that ultrasonic vibration might be originated by slip during the process of decompression of the contacts, while the surface stick during the compressional process [23]. The third mechanism of friction reduction is described by changing the viscoelastic behavior of liquid layer between tip-sample/due to the actuation [24]. It is also suggested that friction is reduced through the extra mechanical energy of actuation in which periodically decrease energy corrugation experienced by the AFM tip [25, 26]. However, these proposed mechanisms have not been investigated quantitatively and in detail, especially for UFM method in which the sample is actuated by a piezoelectric shaker, a few nanometers with amplitude modulation signal at $\mathrm{MHz}$ frequencies.

In this paper, to understand friction reduction in the UFM mode polystyrene (PS) samples are excited at ultrasonic frequencies using amplitude modulation signal. The modulation signal has a ramp shape in which the carrier and modulation frequencies are $1.8 \mathrm{MHz}$ and $2 \mathrm{kHz}$, respectively. Then, by collecting the lateral signal data and calibration of the AFM cantilever the friction forces are evaluated. In both of the CAFM and UFM methods, the friction forces are studied as functions of the normal load, excitation amplitude, and tip radius on PS beads sample. Then, the friction results are investigated on flat film of PS for two different humidites. To investigate and determine the mechanisms of the friction reduction, we applied JKR model for both UFM and CAFM methods in ambient condition and in dry nitrogen. By comparison of the fitting JKR curves with experimental data of C-AFM and UFM, we found that the adhesion forces and shear strength between the tip and the sample are reduced when UFM method is used.

\section{Experimental Methodology}

Figure 1 (a) illustrates the experimental set-up used for all UFM measurements. In this set-up, a commercial AFM system (NanoIR2-ANASYS Instrument Inc.) was retrofitted with an additional piezoelectric actuator (V133-RM Olympus Inc.) mounted below the sample. All experiments were conducted with contact AFM cantilevers (Anasys Instrument Inc.), whose stiffnesses in the normal bending and lateral twisting directions were calibrated using the Sader method [27, 28]. Two different samples were used for experimental studies: thin films of flat PS coated on a silicon substrate and multilayer of PS beads deposited on a silicon substrate. The silicon substrates are cleaned well using ultrasonic bath while immersed in acetone and methanol for 30 minutes to remove any residuals and derbies from the surface. To prepare a thin film of PS, a $10 \mathrm{wt} \%$ solution of PS (Nova 3900, INEOS) dissolved in toluene was spin-coated on silicon substrate at a speed of $4000 \mathrm{rpm}$ for $10 \mathrm{~s}$ followed immediately a second spin cycle at $2000 \mathrm{rpm}$ for $90 \mathrm{~s}$. This results in forming a layer of PS on the silicon substrate of about $300 \mathrm{~nm}$ and a surface roughness of approximately $3 \mathrm{~nm}$. To prepare the multilayer PS bead sample, PS beads of an average diameter of $565 \mathrm{~nm}$ (Thermo Scientific Co.) were deposited from water solution on a cleaned silicon substrate using spin coater at speed of 1000 


\section{Nanotechnology}

(a)

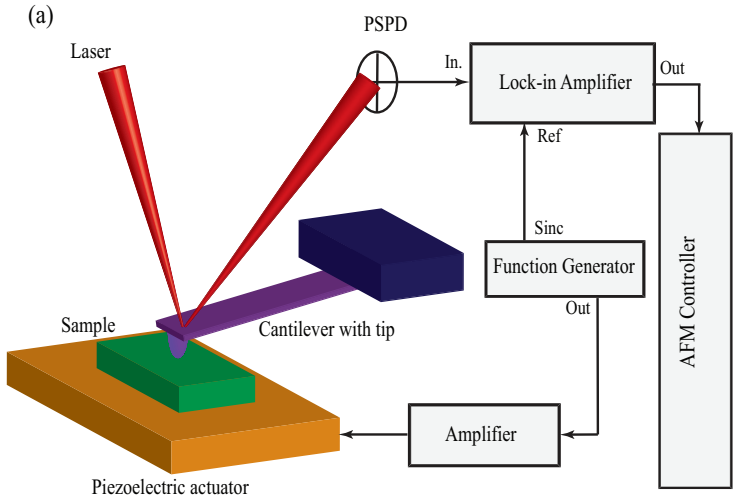

(b)
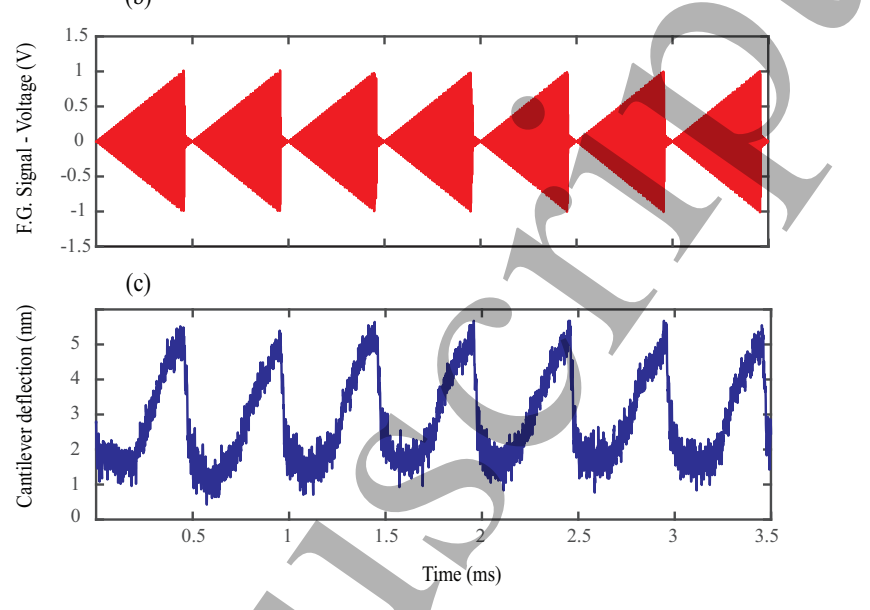

Figure 1. (a) Schematic of UFM set-up. The function generator (F.G.) provides an amplitude modulated signal to the piezoelectric actuator mounted below the sample, actuating the the sample at ultrasonic frequencies. A synchronous output from the function generator provides the reference signal for the lock-in amplifier. The phase and amplitude of the dynamic response of the cantilever at the slower modulation frequency, as measured by the position sensitive photo detector (PSPD), are tracked using a lock-in amplifier for AFM image processing. (b) The ultrasonic excitation signal is modulated in amplitude with a ramp shape in which the carrier and modulation frequencies are $1.8 \mathrm{MHz}$ and $2 \mathrm{kHz}$, respectively. (c) The corresponding cantilever deflection response to modulation signal which was recorded using a data acquisition unit.

rpm for one minute. Following this spin process, the samples were baked at $70^{\circ} \mathrm{C}$ for 40 minutes to increase the adhesion between the polymer and the substrate.

In the UFM mode, samples were excited at ultrasonic frequencies using a piezoelectric actuator beneath the sample [29]. As illustrated in Figure 1 (a), a function generator having an amplitude modulation signal was connected to the piezoelectric actuator, modulating the sample in the $z$-direction (parallel to the normal bending direction of the AFM cantilever) with the same amplitude modulation signal. The induced response of the cantilever by the tip-sample interaction was then detected with a lock-in amplifier. The carrier frequency was much greater than the first resonant frequency of the cantilever (typically $\mathrm{MHz}$ frequencies were used), while the variation of the slower modulation frequency (typically a few $\mathrm{kHz}$ ) was measured by the detection apparatus. The output of lock-in amplifier can also provide information about elastic and adhesive properties of the surface and subsurface features [30, 31] (see supplementary materials for further discussion of this application of UFM).

Figure 1 (b) shows the amplitude modulation signal generated by a function generator in which the carrier and modulation frequencies are $1.8 \mathrm{MHz}$ and $2 \mathrm{kHz}$, respectively. In Figure 1 (c), the AFM cantilever response measured by the PSPD shows the measured out of plane vibration of the sample detected through the deflection of the AFM cantilever. The repetitive jump in the normal deflection of the cantilever is a result of the amplitude modulation of mixed two excitation waves. Except for this modulation 
Nanotechnology

(a)

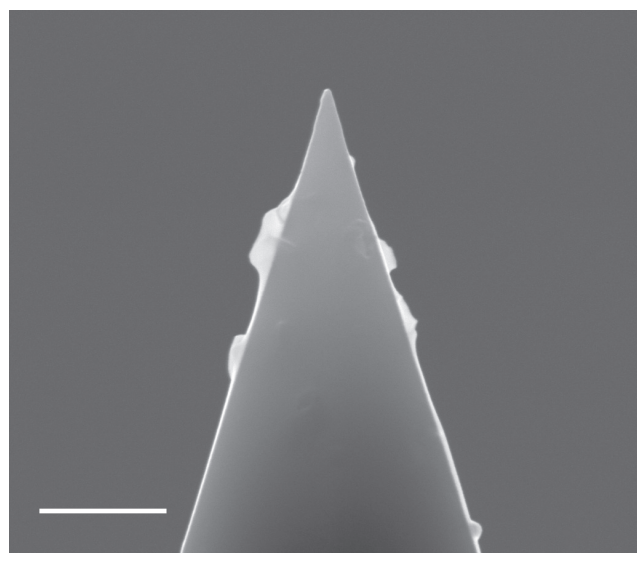

(b)

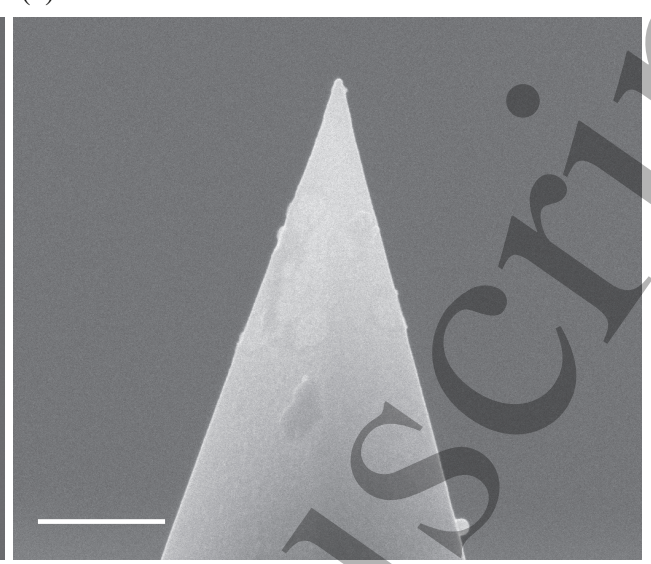

Figure 2. (a) SEM image of the AFM cantilever tip after scanning the flat PS sample. Significant contamination of the tip can be observed by the white contrast at the edges of the pyramidal shaped tip. (b) SEM image of the same tip after cleaning with oxygen plasma. The polymer layer has been removed. The scale bar in both SEM images is 1 $\mu \mathrm{m}$.

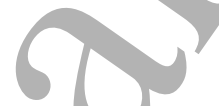

signal, the normal force exerted by the tip on the sample was constant during the scan, as the feedback controller was much slower than measured modulation frequency observed in Figure 1 (c). The friction forces acting between the tip and sample result in the twisting signal of the cantilever, which we refer to as the lateral force. The friction force measured are determined from half the lateral forces measured in the forward direction (trace) subtracted by those measured in the backwards direction (retrace) scans. AFM data were processed using Gwyddion software [32]. The piezoelectric actuator needs to be calibrated accurately to measure the excitation amplitude of the piezoelectric actuator in nm values. The laser Doppler vibrometer (LDV) with very high resolution (15 pm) was used for calibration (see supplementary materials). To determine the radius of the tip before and after scanning, images of the tip apex were captured using a field emission scanning electron microscope (FE-SEM) (Quanta ${ }^{\mathrm{TM}} 250$ FEG). SEM imaging of the tip showed that there was significant contamination of the polymer on the tip after performing friction experiments, as shown in Figure 2 (a). To remove this contamination, AFM tips were cleaned after scanning of polymer samples using oxygen plasma etching for half an hour. Figure 2 (b) shows the result of cleaning the tips with the oxygen plasma.

\section{Results}

In this section, experimental friction results for C-AFM and UFM are presented first for the layer of PS beads deposited on a silicon wafer. In this case, the friction forces are investigated as a function of normal force applied by the AFM tip on the sample followed by the effect of the excitation amplitude of the actuator on friction signals. Additionally, the effect of the tip radius, as observed through two AFM tips with different radii, on 


\section{Nanotechnology}

the measured friction forces under C-AFM and UFM are discussed. Finally, the friction results for the thin films of flat PS will be examined in two different relative humidities (R.H.). This data will be used in next section to develop a theoretical model that can explain the mechanism of the friction reduction in UFM technique.

\subsection{Dependence of friction on the normal force}

Friction as a function of the applied normal force has been measured on the PS beads sample in both UFM and C-AFM modes. In Figure 3, the friction forces were averaged over an entire frame for a given normal force. For those measurements made in the UFM mode, the maximum amplitude of ultrasonic excitation was maintained at a constant value while varying the normal force. For the UFM data in Figure/3, a $5.5 \mathrm{~nm}$ sample excitation amplitude in the out-of-plane direction (see supplementary materials for calibration of piezoelectric actuator) was used. Furthermore, the AFM tip used in this section had a $50 \mathrm{~nm}$ radius. Figure 3 shows a notable increase of the friction with increasing normal force was observed in the C-AFM mode, in particular because of wear at higher loads. Conversely, the trend in friction force with increasing normal force observed for the UFM shows a lower magnitude of friction, as well as a quicker onset to a nearly constant friction at approximately $20 \mathrm{nN}$ normal force compared with 35-40 nN for the C-AFM measurements.

The simultaneously recorded topographic measurements show significant differences in the surface wear or displacement of the PS beads during scanning for the C-AFM and UFM measurements. The topographie measurements have been correlated with loads marked on Figure 3. At point (I), or at low normal forces, the beads remain undisturbed during scanning in both $\mathrm{C}$-AFM and UFM measurements. However, at intermediate loads, marked as (II) in Figure 3, some streaking is observed in the C-AFM measurements, resulting from displacement of some of the PS beads on the surface. However, for the same loads in the UFM measurements, the PS beads remain undisturbed. At high loads, marked as (III) in Figure 3, no structure of the PS bead covered surface can be observed in the C-AFM measurements, indicating significant damage to the organization of the beads on the surface. Contrarily, in UFM, no change in the surface topography of the bead structure is observed. The contrasting behavior of the both the friction force and the observed topographic images of the PS beads during scanning indicates that significant friction reduction between the sliding AFM tip and the surface occurred through the excitation of the sample in the UFM mode, compared with the standard C-AFM measurements. The friction force images of PS beads are shown in supplementary materials.

\subsection{Dependence of friction on the excitation amplitude}

To gain further insight into the mechanisms responsible for the observed reduction in friction when the tip was excited in the UFM mode, the excitation amplitude was varied while friction was measured under three normal forces $(110 \mathrm{nN}, 60 \mathrm{nN}$, and $20 \mathrm{nN})$. 

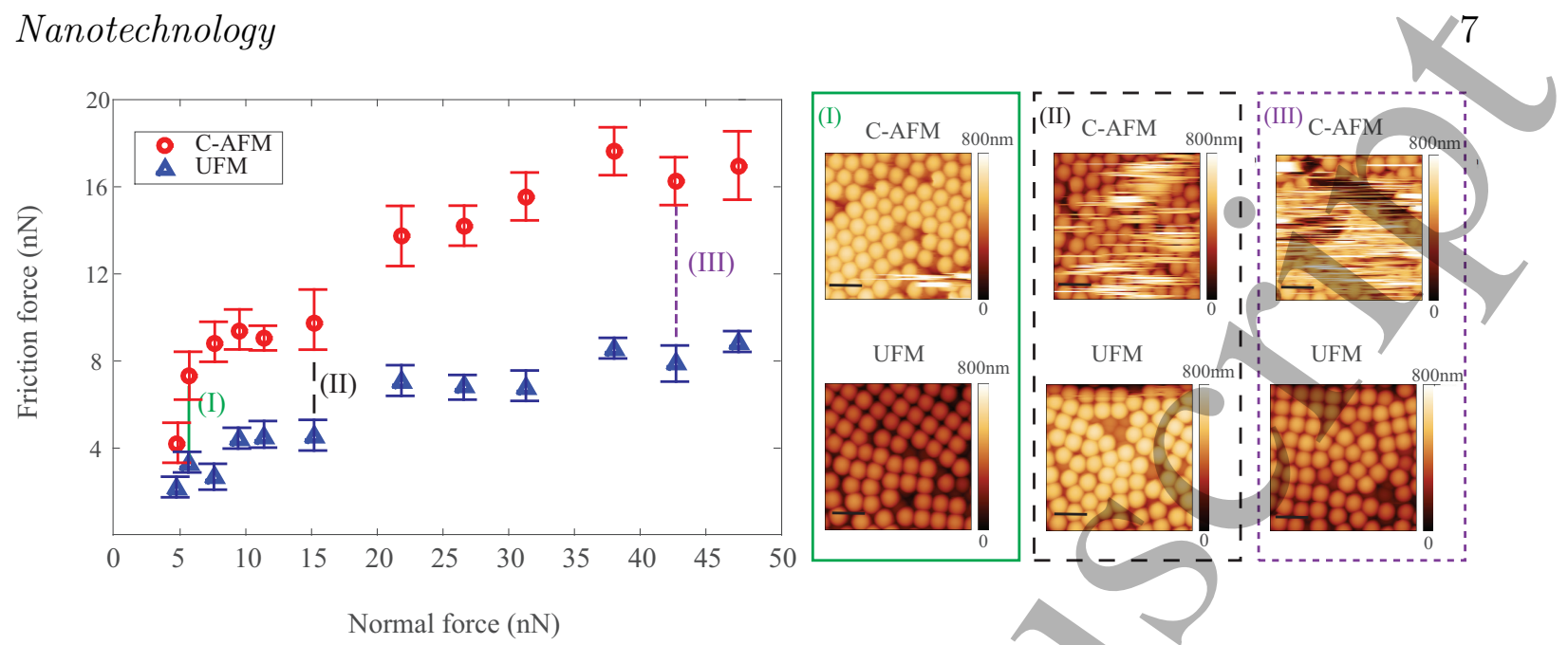

Figure 3. Friction force vs normal force acquired in both C-AFM and UFM modes on the PS bead covered silicon substrate. The friction force versus normal force plot is divided into three regions, marked by a green solid line for region (I) at $6 \mathrm{nN}$, a black dashed line for region (II) at $15 \mathrm{nN}$, and a purple dotted line for region (III) at $42 \mathrm{nN}$. Sample topographic images from both the C-AFM (top) and UFM (bottom) mode scans from each region are encireled with the line corresponding to the region they were taken from. All of the experimental results were collected using an AFM probe having a tip radius of $50 \mathrm{~nm}$. UFM measurements were acquired under a constant excitation amplitude of the $5.5 \mathrm{~nm}$. Finally, measurements were performed under ambient environmental conditions at $10 \%$ relative humidity (R.H.). The lateral scale bars for all topographic images is $1 \mu \mathrm{m}$.

Figure 4 (a) shows that increasing the excitation amplitude reduces friction until the excitation amplitude was increased to approximately $6.5 \mathrm{~nm}$, which further increases in the excitation amplitude did not show further reductions. Three AFM topographic images of the structure of the PS beads are provided in insets of Figure 4 (a) for excitation amplitudes of $4 \mathrm{~nm}, 6.2 \mathrm{~nm}, 7.5 \mathrm{~nm}$ and labeled (I), (II), (III), respectively. These three insets were taken under a constant normal force of $60 \mathrm{nN}$ and with a tip having a $50 \mathrm{~nm}$ radius. The inset labeled (I) shows that with a low excitation amplitude, most of the PS beads are damaged or displaced by the sliding tip due to the high friction forces $(14 \mathrm{nN})$. Compared with the inset labeled (I) in Figure 4 (a), the insets labeled (II) and (II) show that the higher excitation amplitude allowed for the acquisition of topographicimages that resolve the PS bead structure on the surface. In these cases, a significant reduction in friction was observed, with the average friction for the frame in (II) and (III) being $6 \mathrm{nN}$ and $3.5 \mathrm{nN}$, respectively. To understand the non-linear reduction of friction, the deflection signal was acquired at a sufficiently high sampling rate to observe the ultrasonic signal. Figure 4 (b) shows that at low oscillation amplitudes, there is little to no modulation of the deflection signal by the ultrasonic excitation of the sample at amplitudes less than $4 \mathrm{~nm}$. However, once the sample is excited beyond this amplitude, the ultrasonic excitation signal is clearly visible in the deflection signal, and increases in terms of the measured deflection amplitude until the sample is excited at $6 \mathrm{~nm}$ amplitudes. At sample excitation amplitudes beyond $6 \mathrm{~nm}$, no 
Nanotechnology

(a)

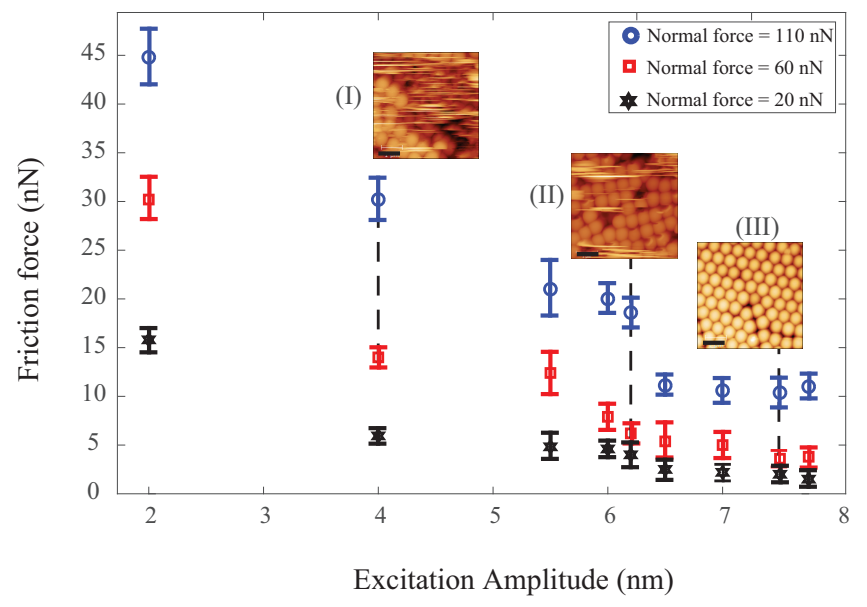

(b)

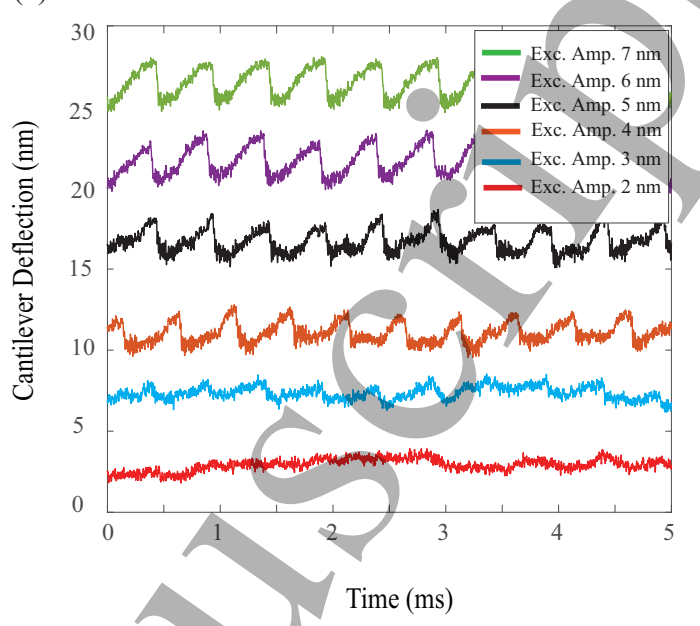

Figure 4. (a) Plots of friction force versus mean excitation amplitude while exciting the sample using the UFM mode. The friction signal was recorded while maintaining a constant average normal force, corresponding to $20 \mathrm{nN}, 60 \mathrm{nN}$ and $110 \mathrm{nN}$. All of the experimental results were collected with a tip radius of $50 \mathrm{~nm}$ in ambient environment with $10 \%$ R.H. Insets labeled (I), (II), (III) show the AFM topography images of PS beads at three different amplitudes and for an applied load of $60 \mathrm{nN}$. Scanning speed was $5 \mu \mathrm{m} / \mathrm{s}$ for all images. The lateral scale bar in all AFM images are $1 \mu \mathrm{m}$.(b) Cantilever deflection signal for different excitation amplitudes. For these measurement, the applied load was $60 \mathrm{nN}$. For clarity, the deflection signal in each excitation amplitude has been offsetted.

change in the cantilever deflection amplitude is observed. This variation in the cantilever deflection signal is strongly linked with the observed trends in friction.

\subsection{Dependence of friction on the tip radius}

Figure 5 shows the impact of tip radius on the measured friction in both C-AFM and UFM modes. Two tips were used in these experiments, one with a tip radius of 25 $\mathrm{nm}$, termed sharp tip in the paper, and $50 \mathrm{~nm}$, referred to later as the blunt tip, on PS beads sample. All experiments in UFM method are performed with a constant excitation amplitude of $7 \mathrm{~nm}$. The SEM images of two tips are given on the right of Figure 5. Figure 5 shows that for the blunt tip the friction force increased notably compared with the sharp tip in C-AFM mode. In comparison, almost no difference in friction was observed for the two tips when friction was measured in the UFM mode. It should be noted when the ultrasonic excitation is applied almost one order of magnitude friction reduction is observed with the blunt tip.

\subsection{Dependence of friction on the humidity}

The examination of the surfaces covered by PS beads have shown that friction and wear are substantially reduced with excitation of the sample at ultrasonic frequencies. In particular, UFM shows the same reduction in friction despite the size of the tip, 

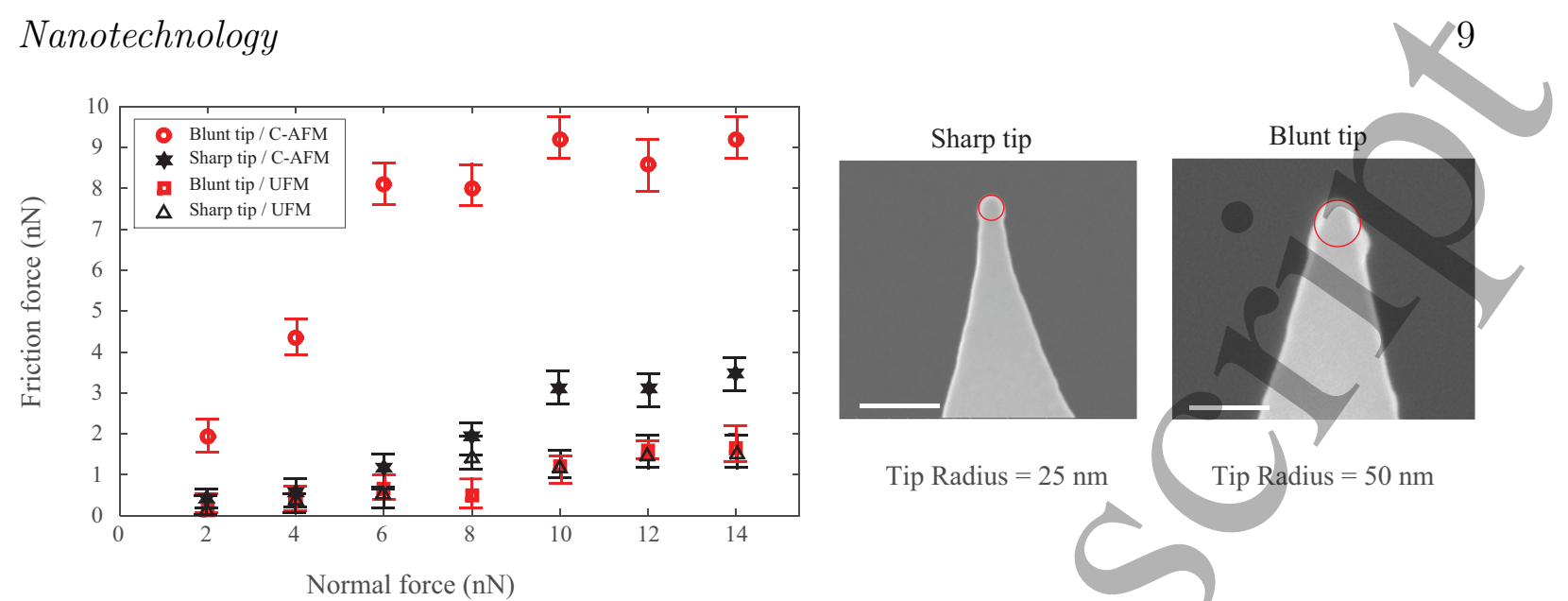

Figure 5. Friction measured on PS beads sample as a function of the normal load for two different tip radius. (b) SEM images of tip radii. The scale bar in both SEM images are $200 \mathrm{~nm}$. All experimental results are collected with constant excitation amplitude of $7 \mathrm{~nm}$.

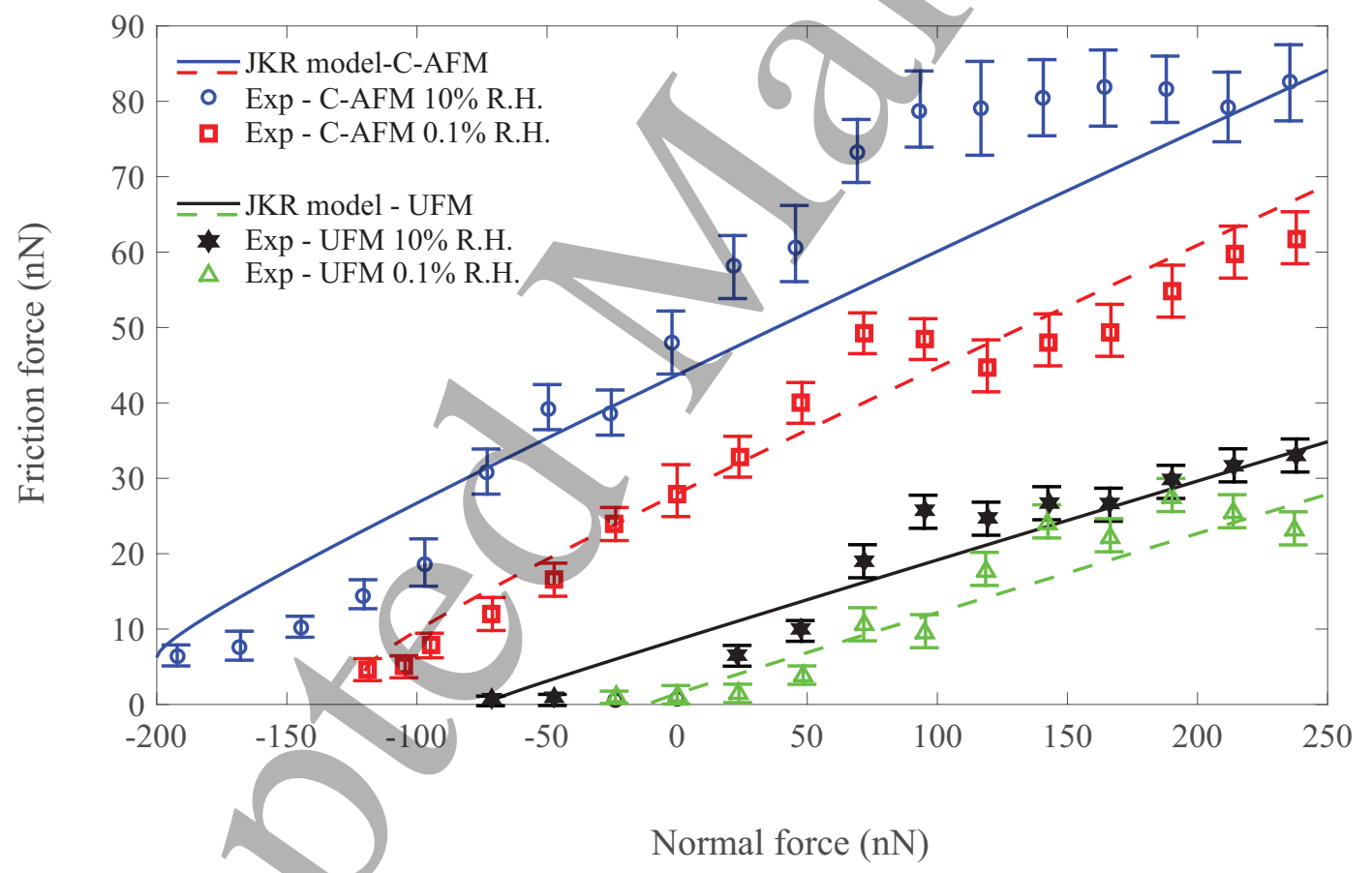

Figure 6. Experimental and Theoretical of friction force vs normal force in both C-AFM and UFM on thin PS film sample. The results were achieved in two different relative humidities (R.H.). The adhesion forces and shear strength have been changed in C-AFM and UFM.

while significant variation in the friction is observed in standard C-AFM scans between the two tips. While the PS beads are an excellent surface, having structure that can be analyzed for wear, the varying topography of the surface may contribute to the observed friction behavior. To isolate the contribution of rough surface topographies to the study of friction, we now examine flat spin-coated PS on silicon wafers, having a thickness of $300 \mathrm{~nm}$ under a humidity controlled environment. 


\section{Nanotechnology}

Table 1. The adhesion forces that are used in calculation of JKR model. These forces are measured using unloading data of friction force vs normal force presented in Figure 6 and those shown in Figure S2.

\begin{tabular}{lcccc}
\hline Measurement & $\begin{array}{c}\text { C-AFM with } \\
10 \% \text { Humidity }\end{array}$ & $\begin{array}{c}\text { C-AFM with } \\
0.1 \% \text { Humidity }\end{array}$ & $\begin{array}{c}\text { UFM with } \\
10 \% \text { Humidity }\end{array}$ & $\begin{array}{c}\text { UFM with } \\
0.1 \% \text { Humidity }\end{array}$ \\
\hline $\begin{array}{l}\text { Pull-off Force } \\
\text { (unloading data) }\end{array}$ & $200 \mathrm{nN}$ & $120 \mathrm{nN}$ & $70 \mathrm{nN}$ & $50 \mathrm{nN}$ \\
$\begin{array}{l}\text { Pull-off Force } \\
\text { (FD curves) }\end{array}$ & $270 \mathrm{nN}$ & $170 \mathrm{nN}$ & $100 \mathrm{nN}$ & $60 \mathrm{nN}$ \\
\hline
\end{tabular}

Figure 6 shows the friction force versus normal force for two humidities $(10 \% \mathrm{RH}$ and $0.1 \% \mathrm{RH}$ ) measured for C-AFM and UFM modes. Similar to previous trends, the UFM mode friction data showed substantially lower friction than the C-AFM data. Furthermore, the experiments conducted under $0.1 \% \mathrm{RH}$ showed lower friction for both the UFM and C-AFM mode studies compared to the same data taken at $10 \% \mathrm{RH}$. These changes of the friction due to the humidity in C-AFM was also discussed using molecular dynamic (MD) simulation [33]. Finally, the experimental data in Figure 6 shows that a significant change in the pull-off force is observed between the two environmental conditions, for both the C-AFM and UFM mode data. This pull-off force data is summarized in Table 1 below. Force vs. distance curves were also acquired for the conditions outlined in Figure 6 and are shown in Figure S2. The values for the pull-off forces are also given in Table 1, showing that both measurements yielded comparable values for all experimental conditions. These results show that for both environmental conditions, use of the UFM mode during friction measurements showed greater than a factor of two decrease in the pull-off force compared with the C-AFM pull-off forces for both humidities. It should be noted that the average friction reduction on flat PS film in UFM method compare to C-AFM is around 60\% (Figure 6). While the average friction reduction on PS beads in UFM method compare to C-AFM is around $50 \%$ (Figure $3)$. Since both of the experiments have been performed in the same conditions, it can be concluded that the contribution of the surface roughness of PS beads on friction reduction is around $10 \%$.

\section{Discussion}

To study the mechanisms of the friction reduction in UFM, the impact on the measured friction on both surfaces, including the PS beads and flat surfaces, under UFM and contact mode are examined. First, we will examine the load-dependent friction behavior measured on the flat PS surface and interpret it using the Johnson Kendall Roberts (JKR) model [34]. In the JKR model, the surface energy per unit area, $\gamma$, is assumed to be confined to the inside of the contact area which is defined by: 
(a)

$$
F_{\text {friction }}=\tau . A
$$

where, $R$ is the radius of curvature of the tip, $F_{\text {applied }}$ is the load applied on tip, and $E^{\star}$ is the modified elastic modulus of the junction. $E^{\star}$ can be determined by:

$$
\frac{1}{E^{\star}}=\frac{1-\nu_{s}^{2}}{E_{s}}+\frac{1-\nu_{t}^{2}}{E_{t}}
$$

where $E_{s}, E_{t}, \nu_{s}$, and $\nu_{t}$ are elastic modulus and Poisson ratio of the sample and the tip, respectively. In the elastic regime, all models for friction assume that the frictional force is proportional to both the real area of contact and the interfacial shear strength as:

where $\tau$ is the shear strength of the polymer. The shear strength of the polymer, $\tau$, is not a constant, but increases linearly with pressure $P$. Many authors have suggested an expression of the form $[35,36,37]$ :

$$
\tau=\tau_{0}+\alpha P
$$




\section{Nanotechnology}

Table 2. Parameters that are used in calculation of $E^{\star}$

\begin{tabular}{lccc}
\hline Material & $\begin{array}{c}\text { Tip radius } \\
(\mathrm{nm})\end{array}$ & Poisson ratio & $\begin{array}{c}\text { Young's modulus } \\
(\mathrm{GPa})\end{array}$ \\
\hline $\mathrm{PS}$ & 50 & 0.35 & 3 \\
$\mathrm{SiO}_{x}$ & - & 0.3 & 165 \\
\hline
\end{tabular}

Table 3. The fitted parameters of shear strength from the C-AFM and UFM mode data using Equation 8 and Figure 6.

\begin{tabular}{cccc}
\hline$\tau_{0}$ & $\alpha$ & $\tau_{0}$ & $\alpha$ \\
C-AFM & C-AFM & UFM & UFM \\
\hline $16 \mathrm{MPa}$ & 0.12 & $4 \mathrm{MPa}$ & 0.1 \\
\hline
\end{tabular}

where $\tau_{0}$ and $\alpha$ are average shear strength of the contact and load coefficient of the shear strength. The adhesive force, $F_{\text {adhesion }}$, acting on the tip is considered as an additional force [35]. Thus, the total normal force, $F_{\text {total }}$, can be mathematically expressed as:

$$
F_{\text {total }}=F_{\text {applied }}+F_{\text {adhesion }}
$$

Therefore:

$$
P=\frac{F_{\text {total }}}{A}
$$

According to the JKR model, the pull-off force, $F_{\text {adhesion, }}$, is related to the work of adhesion as:

$$
F_{\text {adhesion }}=\frac{3}{2} \pi \gamma R \text {. }
$$

By substituting equations 1,4 , and 7 in equation 3 , friction force can be calculated as:

$$
\begin{gathered}
F_{\text {friction }}=\tau_{0} \pi\left(\frac{3 R}{4 E^{\star}}\right)^{\frac{2}{3}}\left(F_{\text {applied }}+2 F_{\text {adhesion }}\right. \\
\left.\left.F_{\text {pplied }}+F_{\text {adhesion }}\right)\right)^{\frac{2}{3}}+\alpha\left(F_{\text {applied }}+F_{\text {adhesion }}\right)
\end{gathered}
$$

Equation 8 can then be used to fit the experimental data shown in Figure 6. Values for the determination of $E^{\star}$ used in the fits are shown in Table 2 and are acquired from Ref. [38] for silicon oxide and Ref. [39, 40] for PS. The adhesive force values used in the fits were taken from Table 1. The data used in the fits are those that were measured from the unloading data of friction force vs. normal force in Figure 6 . These friction force data recorded for both contact AFM and UFM in ambient humidity (10\%) and dry nitrogen $(0.1 \%)$. The fit values determined for the average shear strength are summarized in Table 3. Here we see that, even though the adhesive force used in the fit varied significantly between AFM modes, the average shear strength of the contact was a factor of 4 lower for UFM mode friction scans than for the C-AFM friction scans, and 
the load dependence, $\alpha$ of the shear force is $20 \%$ higher. By comparison of the fitting curves for C-AFM and UFM, it reveals that friction reduction in UFM is mainly due to the reduction of adhesion forces and shear strength of the contact.

The adhesion forces are reduced when the UFM mode is used during friction measurement because of the modulation of the normal force during scanning./The ultrasonic force can be calculated as[41]:

$$
F_{u l t}(d)=\frac{1}{T_{u l t}} \int_{0}^{T_{u l t}} F_{t-s}\left(d_{t-s}-a_{u l t} \cos \left(\frac{2 \pi}{T_{u l t}} t\right)\right) d t
$$

where $d_{t-s}$ is the tip-sample distance, $a_{u l t}$ is the amplitude of ultrasonic vibration, $F_{t-s}$ is applied force on tip, and $T_{u l t}$ is the ultrasonic period. Equation 9 shows that the vertical oscillation of the sample during scanning resulted in a modulation of compressive and tensile forces on the sliding contact. As it was shown in Figure 4 (b), the magnitude of the sample oscillation resulted in a non-linear modulation of the normal force, with little effect at low modulation amplitudes increasing to a modulation of the normal force that did not increase with increasing oscillation of the sample. To interpret the impact of the force modulation on the reduction of adhesion forces, comparison with the nonlinear tip versus sample displacement interaction force is required. Figure 7 (a), shows a sample force distance curve where we assume that tip is in contact with the sample surface, i.e., in the repulsive force regime during a sliding experiment. When the ultrasonic vibration is applied to the sample, tip-sample separation is changed corresponding to the amplitude of the vibration during one period of the modulation signal. When amplitude of vibration is small, it is shown with $a_{0}$, the tip-sample distance sweeps a small section of the curve which is approximately linear. As can be seen in Figure 7 (b), the cantilevers response is equal to the initial set-point force, i.e., the response remains same as in the absence of ultrasonic vibration. However, when the amplitude of ultrasonic vibration is increased to $a_{1}$, the tip-sample separation sweeps into the nonlinear portion of Figure 7 (a). In this case, the cantilevers response experiences an jump deflection that is called ultrasonic deflection or ultrasonic force [29]. We believe that the plateau observed in friction reduction is a result of the tip-sample modulation distance being larger than the linear part of Figure 7 (a).

Additionally, Figure 4(b) shows that UFM modulation of results in a higher timeaveraged normal force. The higher normal force will result in the feedback controller reducing the normal force to maintain the same force set point, compared the set point when there is no oscillation of the sample. This reduction in normal force will result in a decrease in contact area. In the fits shown in Figure 6, no change in contact area between the various modes was assumed. Thus, any changes in the contact area resulting from actuation of the contact will be interpreted in the fits as a change in shear strength, as shown in Table 3. The effect of the ultrasonic frequency parameter on friction reduction is discussed in supplementary materials with the same interpretation.

The reduction of the normal force on the tip due to the ultrasonic vibration can be used to interpret the effect of the tip radius on friction studies. This reduction of 


\section{Nanotechnology}

the normal force in UFM method is mainly affected by ultrasonic parameters such as excitation amplitude and ultrasonic frequency. Therefore, changing the tip radius will not influence on reduction of the normal load on the tip, so as can be seen in Figure 5, we can expect the data do not change significantly in UFM method. To understand the effect of the tip radius on friction forces in C-AFM, we performed our study for applied force $\left(F_{\text {applied }}\right)$ up to $14 \mathrm{nN}$ (Figure 5 ). Since the maximum applied force is much smaller than the adhesion forces $(200 \mathrm{nN})$, we can simplify the contact area in JKR model as:

$$
A=\pi\left(\frac{9 \pi R^{2} \gamma}{2 E^{*}}\right)^{\frac{2}{3}}
$$

Therefore, we can expect 2.5 times increase in friction forces when the tip radius increased two times. The experimental data in Figure 5 showed that the friction forces were increased more than 2.5 times in some applied force. This discrepancy between the JKR model and experimental data might be due to the tip contamination by polymer sample.

Additionally, the reduction in the shear strength when scanning in the UFM mode captured in Table 3 is also a result of energy injected into contact though mechanical excitation of the contact. To consider the influence of the ultrasonic vibration on the quantity of shear strength, $\tau$, we need to investigate the general empirical expression of the shear strength [42]:

$$
\tau=\tau_{0} \ln \left(\frac{V}{h} \cdot \frac{1}{\phi}\right) \exp \left(\frac{-Q}{R T}\right)+\alpha \exp \left(\frac{-V}{d} \cdot \frac{1}{\theta}\right) P
$$

where $V$ is sliding velocity of the tip, $h$ is the polymeric film thickness, $\phi$ and $\theta$ are characteristic frequencies which are functions of temperature and may be thought of as weighted mean values over all the relaxation processes occurring in the polymer, $d$ is the diameter of the contact area, $Q$ is the activation energy, and $T$ is the temperature. As can be seen in Equation 11, the geometry of the contact and the normal load defines the pressure and the contact diameter (and hence the contact time). The effect of the thermal activation and sliding velocity on shear strength, and consequently friction reduction, has already been discussed in detail $[43,44]$.

It should be noted that the wear of the sample can be controlled and reduced when ultrasonic vibration is activated. As it was shown in Figure 3, the wear on PS beads is reduced significantly in UFM method. Similarly to friction, the changes of the shear strength of contact, contact area, and adhesion forces resulted in reduction of sample wear. This demonstration of lowered wear rates on the PS bead sample with UFM suggests of a method to control of the wear on soft samples which are very sensitive to wear such as biological materials. It should be noted that this study is focused on polymer sample where the tip will not be worn quickly. This study could be extended in the future to investigate the effect of the UFM on wear of the tip. This research needs to be performed on a hard material such as silicon or semiconductor where the wear of the tip is a big challenge. 


\section{Conclusion}

The mechanism of friction reduction have been systematically investigated in UFM in comparison with conventional friction force microscopy. In particular, examination of the UFM excitation amplitude, tip radius, normal load on AFM tip, and relative humidity of the environment were examined. Finally, the effects of these parameters on the mechanism of the friction reduction are elaborated using JKR model. It was shown that adhesion forces and shear strength between the tip and the sample are reduced when UFM method is applied. The reduction of these two parameters consequently decrease the friction forces. Our findings may have implications for increasing the efficiency of nanomachining, and nanolithography, and in understanding frictional processes in biological systems. Moreover, the wear on AFM tip and polymeric samples can be controlled to increase their

in imaging and data storage applications.

\section{Acknowledgments}

The authors are very grateful for the support from the Schulich School of Engineering and the Department of Mechanical and Manufacturing Engineering at the University of Calgary, as well as the Natural Sciences and Engineering Research Council of Canada (NSERC) and Alberta Innovates Technology Futures (AITF). SK acknowledges support from the Canada Research Chairs (CRC) program and the Canada Foundation for Innovation (CFI).

\section{References}

[1] M. Urbakh, J. Klafter, D. Gourdon, and J. Israelachvili, "The nonlinear nature of friction," Nature, vol. 430, no. 6999, p. $525,2004$.

[2] Y. Z. K. Lahijania, M. Mohseni, and S. Bastani, "Characterization of mechanical behavior of uv cured urethane acrylate nanocomposite films loaded with silane treated nanosilica by the aid of nanoindentation and nanoscratch experiments," Tribology International, vol. 69, pp. 10-18, 2014.

[3] U. Raviv, S. Giasson, N. Kampf, J.-F. Gohy, R. Jérôme, and J. Klein, "Lubrication by charged polymers," Nature, vol. 425, no. 6954, p. 163, 2003.

[4] C. M. Mate, G. M. McClelland, R. Erlandsson, and S. Chiang, "Atomic-scale friction of a tungsten tip on a graphite surface," in Scanning Tunneling Microscopy, pp. 226-229, Springer, 1987.

[5] J. N. Israelachvili and G. E. Adams, "Measurement of forces between two mica surfaces in aqueous electrolyte solutions in the range 0-100 nm," Journal of the Chemical Society, Faraday Transactions 1: Physical Chemistry in Condensed Phases, vol. 74, pp. 975-1001, 1978.

[6] N. Varongchayakul, S. Johnson, T. Quabili, J. Cappello, H. Ghandehari, S. D. J. Solares, W. Hwang, and J. Seog, "Direct observation of amyloid nucleation under nanomechanical stretching," Acs Nano, vol. 7, no. 9, pp. 7734-7743, 2013.

[7] V. Vahdat and R. W. Carpick, "Practical method to limit tip-sample contact stress and prevent wear in amplitude modulation atomic force microscopy," ACS nano, vol. 7, no. 11, pp. 98369850, 2013. 
Nanotechnology

[8] D. Wong, J. Resendiz, P. Egberts, and S. S. Park, "Reduction of friction using electrospun polymer composite microbeads emulsified in mineral oil," Procedia Manufacturing, vol. 10, pp. 339-350, 2017.

[9] P. Pedraz, R. Wannemacher, and E. Gnecco, "Controlled suppression of wear on the nanoscale by ultrasonic vibrations," ACS nano, vol. 9, no. 9, pp. 8859-8868, 2015.

[10] A. Kovalchenko, O. Ajayi, A. Erdemir, G. Fenske, and I. Etsion, "The effect of laser surface texturing on transitions in lubrication regimes during unidirectional sliding contact," Tribology International, vol. 38, no. 3, pp. 219-225, 2005.

[11] J. Resendiz, P. Egberts, and S. S. Park, "Tribological behavior of multi-scaled patterned surfaces machined through inclined end milling and micro shot blasting," Tribology Letters, vol. 66, no. 4, p. 132, 2018.

[12] S. M. Deese, L. E. Englade-Franklin, L. J. Hill, J. Pyun, J. Y. Chan, and J. C. Garno, "Subsurface imaging of the cores of polymer-encapsulated cobalt nanoparticles using force modulation microscopy," The Journal of Physical Chemistry C, vol. 121, no. 42, pp. 23498-23504, 2017.

[13] M. Cadena, Y. Chen, R. Reifenberger, and A. Raman, "Subsurface imaging using tip generated stress and electric fields in atomic force microscopy," Bulletin of the American Physical Society, 2018.

[14] F. Dinelli, P. Pingue, N. D. Kay, and O. V. Kolosov, "Subsurface imaging of two-dimensional materials at the nanoscale," Nanotechnology, vol. 28, no. 8, p. 085706, 2017.

[15] E. Gnecco, A. Socoliuc, S. Maier, J. Gessler, T. Glatzel, A. Baratoff, and E. Meyer, "Dynamic superlubricity on insulating and conductive surfaces in ultra-high vacuum and ambient environment," Nanotechnology, vol. 20, no. 2, p. 025501, 2008.

[16] F. Dinelli, S. Biswas, G. Briggs, and O. Kolosov, "Ultrasound induced lubricity in microscopic contact," Applied Physics Letters, vol. 71, no. 9, pp. 1177-1179, 1997.

[17] T. Hesjedal and G. Behme, "The origin of ultrasound-induced friction reduction in microscopic mechanical contacts," IEEE transactions on ultrasonics, ferroelectrics, and frequency control, vol. 49, no. 3, pp. 356-364, 2002.

[18] K. Yamanaka, H. Ogiso, and O. Kolosov, "Ultrasonic force microscopy for nanometer resolution subsurface imaging," Applied Physics Letters, vol. 64, no. 2, pp. 178-180, 1994.

[19] B. J. Robinson and O. V. Kolosov, "Probing nanoscale graphene-liquid interfacial interactions via ultrasonic force spectroscopy," Nanoscale, vol. 6, no. 18, pp. 10806-10816, 2014.

[20] M. T. Cuberes and J. J. Martinez, "Mechanical-diode mode ultrasonic friction force microscopy," in Journal of Physics: Conference Series, vol. 61, p. 224, IOP Publishing, 2007.

[21] M. J. Cadena, Y. Chen, R. G. Reifenberger, and A. Raman, "Sub-surface afm imaging using tip generated stress and electric fields," Applied Physics Letters, vol. 110, no. 12, p. 123108, 2017.

[22] H. J. Sharahi, G. Shekhawat, V. Dravid, S. Park, P. Egberts, and S. Kim, "Contrast mechanisms on nanoscale subsurface imaging in ultrasonic afm: scattering of ultrasonic waves and contact stiffness of the tip-sample," Nanoscale, vol. 9, no. 6, pp. 2330-2339, 2017.

[23] S. Dong and M. J. Dapino, "Elastic-plastic cube model for ultrasonic friction reduction via poisson's effect," Ultrasønics, vol. 54, no. 1, pp. 343-350, 2014.

[24] M. T. Cuberes, "Nanoscale friction and ultrasonics," in Fundamentals of Friction and Wear on the Nanoscale, pp. 35-55, Springer, 2015.

[25] U. D. Schwarz and H. Holscher, "Exploring and explaining friction with the prandtl-tomlinson model," ACS nano, vol. 10, no. 1, pp. 38-41, 2016.

[26] A. Socoliuc, E. Gnecco, S. Maier, O. Pfeiffer, A. Baratoff, R. Bennewitz, and E. Meyer, "Atomicscale control of friction by actuation of nanometer-sized contacts," Science, vol. 313, no. 5784, pp. 207-210, 2006.

[27] J. E. Sader, J. W. Chon, and P. Mulvaney, "Calibration of rectangular atomic force microscope cantilevers," Review of Scientific Instruments, vol. 70, no. 10, pp. 3967-3969, 1999.

[28] C. P. Green, H. Lioe, J. P. Cleveland, R. Proksch, P. Mulvaney, and J. E. Sader, "Normal and torsional spring constants of atomic force microscope cantilevers," Review of Scientific 
1

2

3

4

5

6

7

8

9
Nanotechnology

Instruments, vol. 75, no. 6, pp. 1988-1996, 2004.

[29] K. Yamanaka, "Ultrasonic force microscopy," MRS bulletin, vol. 21, no. 10, pp. 36-41, 1996.

[30] M. H. van Es, A. Mohtashami, R. M. Thijssen, D. Piras, P. L. van Neer, and H. Sadeghian, "Mapping buried nanostructures using subsurface ultrasonic resonance force microscopy," Ultramicroscopy, vol. 184, pp. 209-216, 2018.

[31] Y. Sun, L. Tian, J. Wen, J. Zhao, W. Zhang, C. Xie, M. Zhou, X. Qiu, and D. Chen, "Morphologies of eumelanins from the ink of six cephalopods species measured by atomic force microscopy," Journal of Ocean University of China, vol. 16, no. 3, pp. 461-467, 2017.

[32] D. Nečas and P. Klapetek, "Gwyddion: an open-source software for spm data analysis," Open Physics, vol. 10, no. 1, pp. 181-188, 2012.

[33] J. Shi, J. Chen, L. Fang, K. Sun, J. Sun, and J. Han, "Atomistic scale nanoscratching behavior of monocrystalline cu influenced by water film in cmp process," Applied Surface Science, vol. 435, pp. 983-992, 2018.

[34] K. L. Johnson, K. Kendall, and A. Roberts, "Surface energy and the contact of elastic solids," Proc. R. Soc. Lond. A, vol. 324, no. 1558, pp. 301-313, 1971.

[35] D. Gracias and G. Somorjai, "Continuum force microscopy study of the elastic modulus, hardness and friction of polyethylene and polypropylene surfaces," Macromolecules, vol. 31, no. 4, pp. 1269-1276, 1998.

[36] R. W. Carpick, D. F. Ogletree, and M. Salmeron, "A general equation for fitting contact area and friction vs load measurements," Journal of colloid and interface science, vol. 211, no. 2, pp. 395-400, 1999.

[37] X. Wang, O. K. C. Tsui, and X. Xiao, "Dynamic study of polymer films by friction force microscopy with continuously varying load," Langmuir, vol. 18, no. 18, pp. 7066-7072, 2002.

[38] J. P. Killgore, J. Y. Kelly, C. M. Stafford, M. J. Fasolka, and D. C. Hurley, "Quantitative subsurface contact resonance force microscopy of model polymer nanocomposites," Nanotechnology, vol. 22, no. 17, p. 175706, 2011.

[39] M. E. Dokukin and I. Sokolov, "Quantitative mapping of the elastic modulus of soft materials with harmonix and peakforce qnm afm modes," Langmuir, vol. 28, no. 46, pp. 16060-16071, 2012.

[40] K. Miyake, N. Satomi, and S. Sasaki, "Elastic modulus of polystyrene film from near surface to bulk measured by nanoindentation using atomic force microscopy," Applied physics letters, vol. 89, no. 3, p. 031925, 2006.

[41] A. Briggs, G. Briggs, and O. Kolosov, Acoustic microscopy, vol. 67. Oxford University Press, 2010.

[42] B. Briscoe and D. Tabor, "Shear properties of thin polymeric films," The Journal of Adhesion, vol. 9, no. 2, pp. 145-155, 1978 .

[43] C. Greiner, J. R. Felts, Z. Dai, W. P. King, and R. W. Carpick, "Controlling nanoscale friction through the competition between capillary adsorption and thermally activated sliding," $A C S$ nano, vol. 6, no. 5, pp. 4305-4313, 2012.

[44] Y. Dong, H. Gao, A. Martini, and P. Egberts, "Reinterpretation of velocity-dependent atomic friction: Influence of the inherent instrumental noise in friction force microscopes," Physical Review E, vol. 90, no. 1, p. 012125, 2014. 